\title{
ROBUSTNESS WITH RESPECT TO SMALL DELAYS FOR EXPONENTIAL STABILITY OF ABSTRACT DIFFERENTIAL EQUATIONS IN BANACH SPACES
}

\author{
FAMING GUO $^{\ominus 1}$, BIN TANG ${ }^{2}$ and FALUN HUANG ${ }^{3}$
}

(Received 17 January, 2004; revised 22 February, 2006)

\begin{abstract}
This paper is concerned with robustness with respect to small delays for the exponential stability of abstract differential equations in Banach spaces. Some necessary and sufficient conditions are given in terms of the uniformly square integrability of the fundamental operator family and the uniform boundedness of its resolvent on the imaginary axis.

2000 Mathematics subject classification: primary 93C20; secondary 35B40.

Keywords and phrases: small delay, robust stability, fundamental operator.
\end{abstract}

\section{Introduction and Preliminaries}

Robustness of stability with respect to small delays, for example, as motivated by feedback systems in control theory, is of great theoretical and practical importance, but this property does not hold for many systems. In the literature (see, for example, $[4,5,7,9,10]$ and references therein), there are many examples of systems described by distributed parameters which are exponentially stabilised by a feedback but which are destabilised by arbitrary small delays in the feedback loop. Examples of this sort first appeared in Huang [9] and Datko et al. [5] independently in 1986. Huang [9] gave two abstract counterexamples and proved a well-known result that a system is robust to small delays if operator $A$ generates an immediately norm continuous $C_{0}$-semigroup. More recently, the problem of robustness of stability has received considerable attention. Logemann, Rebarber, Townley and Weiss ([13-16]) have presented a systematic

\footnotetext{
'School of Applied Mathematics and School of Electronic Engineering, University of Electronic Science and Technology of China, Chengdu 610054, P. R. China; e-mail: guofm@uestc.edu.cn. ${ }^{2}$ School of Electronic Engineering, University of Electronic Science and Technology of China, Chengdu 610054, P. R. China.

${ }^{3}$ Mathematical College, Sichuan University, Chengdu 610064, P. R. China.

(C) Australian Mathematical Society 2006, Serial-fee code 1446-1811/06
} 
treatment for some distributed parameter systems. More recently, Avalos et al. [1] showed that it is not possible to construct a dynamic stabiliser of a general form such that the stabilisation is robust with respect to small delays for a structural acoustics model. Hale and Lunel [7] try to explain the underlying mechanisms and the role that difference equations are playing in robustness results. Batkai and Piazzera [2] show that those sufficient conditions derived in [10] and [14] also hold on the state space $\mathcal{E}=X \times L^{p}\left(\left[0, r_{0}\right], X\right)$, which is a better choice for many applications.

Corduneanu [3] first introduced the fundamental matrix to study stability for integro-differential equations in finite-dimensional spaces. Liang and Xiao ([11] and [12]) extended the fundamental matrix to the fundamental operator to deal with exponential stability for abstract autonomous functional differential equations with infinite delay. In this paper we investigate whether exponential stability persists if there is a small delay in the feedback term for the abstract differential equation by using the fundamental operator family in Banach spaces.

Consider the following equation:

$$
\begin{cases}\dot{u}(t)=A u(t)+B u(t-r), & t>0, \\ u(0)=x, & \theta \in[-r, 0]\end{cases}
$$

on a Banach space $X$, where $(A, D(A))$ generates a $C_{0}$-semigroup $T(t)$ satisfying $\|T(t)\| \leq M e^{w t}, w \in R, M \geq 1 ; B \in \mathcal{L}(X), x_{0} \in X, f \in L^{p}([-r, 0] ; X), p \geq 1$. For concepts and results involving the $C_{0}$-semigroup, we refer the reader to [6, 8] and [18].

To transform system (1.1) into operator-valued matrix form we introduce the following operator $\mathcal{A}_{r}$ on $\mathcal{E}:=X \times L^{p}([-r, 0] ; X)$ :

$$
\begin{aligned}
\mathcal{A}_{r} & =\left(\begin{array}{cc}
A & B \delta_{-r} \\
0 & d / d \sigma
\end{array}\right), \\
D\left(\mathcal{A}_{r}\right) & =\left\{(x, f) \in D(A) \times W^{1 . p}([-r, 0] ; X): f(0)=x\right\},
\end{aligned}
$$

and system (1.1) is transformed into the equivalent Cauchy problem on $\mathcal{E}$ :

$$
\left\{\begin{array}{l}
\dot{v}(t)=\mathcal{A}_{r} v(t) \\
v(0)=v_{0}
\end{array}\right.
$$

Under these assumptions on $A$ and $B,(A+B, D(A))$ generates a $C_{0}$-semigroup. It is easy to prove that $\left(\mathcal{A}_{r}, D\left(\mathcal{A}_{r}\right)\right)$ generates a $C_{0}$-semigroup $\mathcal{T}_{r}(t)=\left(\begin{array}{l}u(t) \\ { }_{u_{t}(\cdot)}\end{array}\right)$ on $\mathcal{E}$. For details, we refer to [2].

In the following section, we introduce the fundamental operator family and obtain a representation of the solution of system (1.1). In Section 3, some necessary and sufficient conditions are given in terms of the uniformly square integrability of the fundamental operator family and the uniform boundedness of its resolvent on the imaginary axis. 


\section{Preliminaries}

To characterise robustness with respect to small delays for the exponential stability of system (1.1) on the state space $\mathcal{E}$, we introduce the concept of the fundamental operator family for system (1.1) by means of the following integral equation:

$$
\begin{cases}X_{r}(t) x=T(t) x+\int_{0}^{t} T(t-s) B X_{r}(s-r) x d s, & t>0, \\ X_{r}(0)=I, & t=0, \\ X_{r}(t)=0, & t \in[-r, 0) .\end{cases}
$$

In this section, we first derive the existence of the strong continuous solution $X_{r}(t), t \geq 0$, and an exponential estimate on $X_{r}(t)$ which are basic to the application of the Laplace transform and to obtaining a representation of the solution $u(t)$ of system (1.1). It is easy to prove the following theorem.

THEOREM 2.1. Suppose that the conditions on $A$ and $B$ are satisfied. Then there exists a unique solution $X_{r}(t), t \geq-r$, of Equation (2.1) which is strongly continuous on $[0, \infty)$. Furthermore, $X_{r}(t)$ satisfies the inequality

$$
\left\|X_{r}(t) x\right\| \leq M e^{\left(w+M e^{|\omega| r}\|B\|\right) t}\|x\|, \quad t \geq r .
$$

From Theorem 2.1, we can define the fundamental operator family $X_{r}(t), t \geq-r$, as the unique solution of integral equation (2.1). From $\left\|X_{r}(t)\right\| \leq M e^{w_{1} t}$ (where $\left.w_{1}=w+M e^{|w| r}\|B\|\right)$, we obtain that the integral $\int_{0}^{\infty} e^{-\lambda t} X_{r}(t) x d t$ converges for all $\operatorname{Re} \lambda>w_{1}$ and all $x \in X$. Furthermore, we have the following theorem which implies that $\Delta_{r}^{-1}(\lambda):=\left(\lambda-A-e^{-\lambda r} B\right)^{-1}$ is the Laplace transform of $X_{r}(t)$.

THEOREM 2.2. The fundamental operator family $X_{r}(t)$ of Equation (1.1) satisfies

$$
\int_{0}^{\infty} e^{-\lambda s} X_{r}(t) x d t=\Delta_{r}^{-1}(\lambda) x, \quad \operatorname{Re} \lambda>w_{1}>w \text { for } x \in X
$$

Proof. Since $X_{r}(t)$ satisfies

$$
X_{r}(t) x=T(t) x+\int_{0}^{t} T(t-s) B X_{r}(s-r) x d s, \quad t \geq 0 \text { and } x \in X,
$$

we have

$$
\int_{0}^{\infty} e^{-\lambda t} X_{r}(t) x d t=\int_{0}^{\infty} e^{-\lambda t} T(t) x d t+\int_{0}^{\infty} e^{-\lambda t} \int_{0}^{t} T(t-s) B X_{r}(s-r) x d s d t
$$




$$
\begin{aligned}
& =R(\lambda, A) x+\int_{0}^{\infty} \int_{0}^{\infty} e^{-\lambda(t+s)} T(t) B X_{r}(s-r) x d t d s \\
& =R(\lambda, A) x+\int_{0}^{\infty} R(\lambda, A) e^{-\lambda s} B X_{r}(s-r) x d s \\
& =R(\lambda, A) x+\int_{r}^{\infty} R(\lambda, A) e^{-\lambda s} B X_{r}(s-r) x d s \\
& =R(\lambda, A) x+e^{-\lambda r} R(\lambda, A) B \int_{0}^{\infty} e^{-\lambda s} X_{r}(s) x d s .
\end{aligned}
$$

Therefore

$$
\left(\lambda-A-B e^{-\lambda r}\right) \int_{0}^{\infty} e^{-\lambda t} X_{r}(t) x d t=x
$$

which yields that

$$
\int_{0}^{\infty} e^{-\lambda t} X_{r}(t) x d t=\Delta_{r}^{-1}(\lambda) x,
$$

for $\operatorname{Re} \lambda$ large enough, and hence it holds for $\operatorname{Re} \lambda>w_{1}$.

THEOREM 2.3. Let $u(t)$ be the mild solution of (1.1). Then $u(t)$ is given by

$$
u(t)= \begin{cases}X_{r}(t) x_{0}+\int_{0}^{t} X_{r}(t-s) B\left(S_{0} f\right)(s-r) d s, & t \geq 0, \\ f(t), & t \in[-r, 0),\end{cases}
$$

where

$$
\left(S_{0} f\right)(t)= \begin{cases}f(t), & t \in[-r, 0) \\ 0, & \text { otherwise }\end{cases}
$$

Proof. (a) Since $X_{r}(t)$ satisfies (2.1), for $t \in[0, r]$ we have

$$
\begin{aligned}
X_{r}(t) & =T(t) \text { and } \\
\int_{0}^{t} X_{r}(t-s) B\left(S_{0} f\right)(s-r) d s & =\int_{0}^{t} T(t-s) B f(s-r) d s,
\end{aligned}
$$

and consequently,

$$
\begin{aligned}
u(t) & =T(t) x_{0}+\int_{0}^{t} T(t-s) B u(s-r) d s \\
& =X_{r}(t) x_{0}+\int_{0}^{t} X_{r}(t-s) B f(s-r) d s \\
& =X_{r}(t) x_{0}+\int_{0}^{t} X_{r}(t-s) B\left(S_{0} f\right)(s-r) d s .
\end{aligned}
$$


For $t \in[r, 2 r)$, from (2.1) it follows that

$$
\begin{aligned}
X_{r}(t) x_{0} & =T(t) x_{0}+\int_{0}^{t} T(t-s) B X_{r}(s-r) x_{0} d s \\
& =T(t) x_{0}+\int_{0}^{t} T(t-s) B(s-r) x_{0} d s,
\end{aligned}
$$

and

$$
\begin{aligned}
\int_{0}^{t} X_{r}(t-s) B\left(S_{0} f\right)(s-r) d s \\
=\int_{0}^{r} T(t-s) B f(s-r) d s \\
\quad+\int_{0}^{r} \int_{0}^{t-s} T(t-s-\tau) B X_{r}(\tau-r) B f(s-r) d \tau d s \\
=\int_{0}^{r} T(t-s) B f(s-r) d s \\
\quad+\left(\int_{r}^{t} \int_{0}^{r}+\int_{0}^{r} \int_{0}^{s}\right) T(t-s) B X_{r}(s-\tau-r) B f(s-r) d \tau d s \\
=\int_{0}^{r} T(t-s) B f(s-r) d s \\
\quad+\int_{r}^{t} \int_{0}^{s-r} T(t-s) B X_{r}(s-\tau-r) B f(s-r) d \tau d s .
\end{aligned}
$$

Using (2.3), (2.4), and the fact that $u(t)$ is the mild solution of (1.1), we deduce that

$$
\begin{aligned}
u(t)= & T(t) x_{0}+\int_{r}^{t} T(t-s) B u(s-r) d s+\int_{0}^{r} T(t-s) B f(s-r) d s \\
= & T(t) x_{0}+\int_{r}^{t} T(t-s) B T(s-r) x_{0} d s+\int_{0}^{r} T(t-s) B f(s-r) d s \\
& +\int_{0}^{r} \int_{0}^{s-r} T(t-s) B T(s-r-\tau) B f(\tau-r) d \tau d s \\
= & X_{r}(t) x_{0}+\int_{0}^{t} X_{r}(t-s) B\left(S_{0} f\right)(s-r) d s .
\end{aligned}
$$

(b) Suppose that (2.2) holds for $t \in[0,(n+1) r)$. Now for $t \in[(n+1) r,(n+2) r)$, we have

$$
\begin{aligned}
u(t) & =T(t) x_{0}+\int_{r}^{t} T(t-s) B u(s-r) d s+\int_{0}^{r} T(t-s) B f(s-r) d s \\
& =T(t) x_{0}+\int_{r}^{t} T(t-s) B X_{r}(s-r) x_{0} d s
\end{aligned}
$$




$$
\begin{aligned}
& +\int_{r}^{t} \int_{0}^{\tau-r} T(t-\tau) B X_{r}(\tau-r-s) B\left(S_{0} f\right)(s-r) d s d \tau \\
+ & \int_{0}^{r} T(t-s) B f(s-r) d s+\int_{0}^{r} T(t-s) B f(s-r) d s \\
= & X_{r}(t) x_{0}+\int_{0}^{r} \int_{r}^{t-s} T(t-s-\tau) B X_{r}(\tau-r) B f(s-r) d \tau d s \\
& +\int_{0}^{r} T(t-s) B f(s-r) d s,
\end{aligned}
$$

and

$$
\begin{aligned}
\int_{0}^{t} X_{r}(t-s) B\left(S_{0} f\right)(s-r) d s \\
\quad=\int_{0}^{t} T(t-s) B f(s-r) d s \\
\quad+\int_{0}^{r} \int_{r}^{t-s} T(t-s-\tau) B X_{r}(\tau-r) B f(s-r) d \tau d s
\end{aligned}
$$

From (2.5) and (2.6), it follows that

$$
u(t)=X_{r}(t) x_{0}+\int_{0}^{t} X_{r}(t-s) B\left(S_{0} f\right)(s-r) d s, \quad t \in[(n+1) r,(n+2) r),
$$

which completes the proof of the theorem.

\section{Robustness with respect to small delays}

In this section, we first introduce the concept of robustness with respect to small delays for exponential stability on the state space $\mathcal{E}=X \times L^{p}([-r, 0] ; X)$. Secondly, the robustness is characterised via the fundamental operators. Finally, we show the analyticity of the resolvent $\Delta_{r}^{-1}(\lambda)$ and the resolvent identity, and furthermore, characterise the robustness in terms of the resolvent $\Delta_{r}^{-1}(\lambda)$.

DEFINITION 1. System (1.1) is said to be robust with respect to small delays for exponential stability if there exists $r_{0}>0$ such that for any $r \in\left[0, r_{0}\right], f \in L^{p}([-r, 0] ; X)$ and $x \in X$, system (1.1) is exponentially stable, that is, there exist constants $M, w>0$ such that

$$
\left\|\mathcal{T}(t)\left(\begin{array}{c}
x \\
f
\end{array}\right)\right\|_{\mathcal{E}}=\left\|\left(\begin{array}{c}
u(t) \\
u_{t}
\end{array}\right)\right\|_{\mathcal{E}} \leq M e^{-w r}\left(\|x\|^{p}+\|f\|_{L^{p}([-r, 0] ; x)}^{p}\right)^{l / p} .
$$


THEOREM 3.1. System (1.1) is robust with respect to small delays for exponential stability if and only if there exists $r_{0}>0$ such that $X_{r}(t)$ is uniformly exponentially stable for $r \in\left[0, r_{0}\right]$, that is, there exist constants $r_{0}, M, w>0$ such that

$$
\left\|X_{r}(t)\right\| \leq M e^{-w r}, \quad t \geq 0, \text { uniformly for } r \in\left[0, r_{0}\right] .
$$

PROOF. For any $x \in X$, let $f=0$. We deduce that the mild solution $u(t)$ of (1.1) satisfies

$$
u(t)= \begin{cases}X_{r}(t) x, & t \geq 0, \\ 0, & t \in[-r, 0) .\end{cases}
$$

Therefore, robustness shows that $\|u(t)\|=\left\|X_{r}(t) x\right\| \leq M e^{-w t}\|x\|, x \in X$, which implies $\left\|X_{r}(t)\right\| \leq M e^{-w t}$ uniformly for $r \in\left[0, r_{0}\right]$.

On the other hand, for $t>r$, noticing that $B\left(S_{0} f\right)(s-r)=0, s>r$, and

$$
u(t)=X_{r}(t) x+\int_{0}^{t} X_{r}(t-s) B\left(S_{0} f\right)(s-r) d s,
$$

we get

$$
\begin{aligned}
\|u(t)\| & \leq M e^{-w t}\|x\|+\int_{0}^{t} M e^{-w(t-s)}\|B\|\|f(s-r)\| d s \\
& \leq M e^{-w t}\|x\|+M\|B\| e^{-w t}\left(\frac{1}{q w}\left(e^{q w r_{0}}-1\right)\right)^{1 / q}\|f\|_{L^{\rho}([-r, 0] ; X)} \\
& \leq M^{\prime} e^{-w t}\left(\|x\|+\|f\|_{L^{\rho}([-r, 0] ; X)}\right) \\
& \leq M^{\prime} e^{-w t} 2\left(\|x\|^{p}+\|f\|_{L^{2}([-r, 0] ; X)}^{p}\right)^{1 / p},
\end{aligned}
$$

where $1 / p+1 / q=1$. Similarly, we have

$$
\|u(t+\theta)\| \leq M e^{-w t} e^{-w \theta}\|x\|+\int_{0}^{r} M e^{-w t} e^{w s} e^{-w \theta}\|B\|\|f(s-r)\| d s,
$$

and consequently,

$$
\begin{aligned}
\left\|u_{t}(\cdot)\right\|_{L^{p}([-r, 0] ; X)} \leq & M\left(\frac{1}{p w}\left(e^{p w r_{0}}-1\right)\right)^{1 / p} e^{-w t}\|x\| \\
& +M e^{-w t}\|B\|\left(\frac{1}{p w}\left(e^{p w r_{0}}-1\right)\right)^{1 / p} \int_{0}^{r} e^{w s} f(s-r) d s \\
\leq & 2 M^{\prime \prime} e^{-w t}\left(\|x\|^{p}+\|f\|_{L^{p}([-r, 0]: X)}^{p}\right)^{1 / p} .
\end{aligned}
$$

From the above discussion, we deduce that

$$
\left\|\mathcal{T}(t)\left(\begin{array}{l}
x \\
f
\end{array}\right)\right\|_{\mathcal{E}}=\left\|\left(\begin{array}{c}
u(t) \\
u_{t}(\cdot)
\end{array}\right)\right\|_{\mathcal{E}} \leq 2 \max \left\{M^{\prime}, M^{\prime \prime}\right\} e^{-w t}\left(\|x\|^{p}+\|f\|_{\left.L^{p}(1-r, 0] ; x\right)}^{p}\right)^{1 / p},
$$

which implies robustness. 
THEOREM 3.2. $X_{r}(t)$ is uniformly exponentially stable for $r \in\left[0, r_{0}\right]$ if and only if there exists $M>0$ such that

$$
\left(\int_{0}^{\infty}\left\|X_{r}(t) x\right\|^{2} d t\right)^{1 / 2} \leq M\|x\|, \quad \text { for } x \in X \text { and uniformly for } r \in\left[0, r_{0}\right] .
$$

PROOF. It suffices to show sufficiency for $p=2$. Indeed, if $u(t)$ is the mild solution of (1.1), then for $t>0$, we have

$$
u(t)=X_{r}(t) x+\int_{0}^{t} X_{r}(t-s) B\left(S_{0} f\right)(s-r) d s,
$$

which implies

$$
\begin{aligned}
\|u(\cdot)\|_{L^{2}(0, \infty ; X)} \leq & \left\|X_{r}(\cdot) x\right\|_{L^{2}(0, \infty ; X)} \\
& +\left\{\int_{0}^{\infty}\left(\int_{0}^{t}\left\|X_{r}(t-s) B\left(S_{0} f\right)(s-r)\right\| d s\right)^{2} d t\right\}^{1 / 2} \\
\leq & M\|x\|+\int_{0}^{\infty}\left(\int_{0}^{\infty}\left\|X_{r}(t-s) B\left(S_{0} f\right)(s-r)\right\|^{2} d t\right)^{1 / 2} d s \\
\leq & M\|x\|+\int_{0}^{\infty} M\left\|B\left(S_{0} f\right)(s-r)\right\| d s \\
\leq & M^{\prime}\left(\|x\|^{2}+\|f\|_{L^{2}([-r, 0] ; X)}^{2}\right)^{1 / 2} .
\end{aligned}
$$

On the other hand, for $t>r$, we can deduce

$$
\begin{aligned}
\left(\int_{r}^{\infty}\right. & \left.\int_{-r}^{0}\left\|u_{t}(\theta)\right\|^{2} d \theta d t\right)^{1 / 2} \\
\leq & \left(\int_{0}^{\infty} \int_{-r}^{0}\left\|X_{r}(t+\theta) x\right\|^{2} d \theta d t\right)^{1 / 2} \\
& +\left(\int_{r}^{\infty} \int_{-r}^{0}\left(\int_{0}^{t+\theta}\left\|X_{r}(t+\theta-s) B\left(S_{0} f\right)(s-r)\right\| d s\right)^{2} d \theta d t\right)^{1 / 2} \\
\leq & \left(\int_{-r}^{0} \int_{r}^{\infty}\left\|X_{r}(t+\theta) x\right\|^{2} d t d \theta\right)^{1 / 2} \\
& +\left(\int_{-r}^{0} \int_{0}^{\infty} \int_{r}^{\infty}\left\|X_{r}(t+\theta-s) B\left(S_{0} f\right)(s-r)\right\|^{2} d t d s d \theta\right)^{1 / 2} \\
\leq & M r\|x\|+\left(\int_{-r}^{0} \int_{0}^{\infty} M\left\|B\left(S_{0} f\right)(s-r)\right\|^{2} d s d \theta\right)^{1 / 2} \\
\leq & M^{\prime \prime}\left(\|x\|+\|f\|_{L^{2}(\{-r, 0\} ; X)}\right) .
\end{aligned}
$$


From (3.1) and (3.2), it follows that

$$
\left(\int_{0}^{\infty}\left\|\mathcal{T}(t)\left(\begin{array}{l}
x \\
f
\end{array}\right)\right\|_{\mathcal{E}}^{2} d t\right)^{1 / 2}=\left(\int_{0}^{\infty}\left\|\left(\begin{array}{l}
u(t) \\
u_{t}(\cdot)
\end{array}\right)\right\|_{X \times L^{\rho}([-r, 0] ; X)}^{2} d t\right)^{1 / 2}<+\infty
$$

By Datko-Pazy's theorem $([6,18])$ for exponential stability, we obtain that $\mathcal{T}(t)$ is exponentially stable which implies that $X_{r}(t)$ is uniformly exponentially stable for $r \in\left[0, r_{0}\right]$.

In what follows, suppose that $D_{r}:=\left\{\lambda \in C \mid \Delta_{r}^{-1}(\lambda)\right.$ exists and $\left.\Delta_{r}^{-1}(\lambda) \in \mathcal{L}(X)\right\}$. We will show the $\mathcal{L}(X)$-valued analyticity and the resolvent identity of $\Delta_{r}^{-1}(\lambda)$ on $D_{r}$, and characterise the robustness of the stability by $\Delta_{r}^{-1}(\lambda)$.

THEOREM 3.3. (i) For all $\mu, \lambda \in D_{r}$, we have the following resolvent identity:

$$
\Delta_{r}^{-1}(\mu)-\Delta_{r}^{-1}(\lambda)=\Delta_{r}^{-1}(\mu)\left(\lambda-\mu+B e^{-\mu r}-B e^{-\lambda r}\right) \Delta_{r}^{-1}(\lambda) .
$$

(ii) The resolvent $\Delta_{r}^{-1}(\cdot)$ is analytic on $D_{r}$, and

$$
\frac{d}{d \lambda} \Delta_{r}^{-1}(\lambda)=-\Delta_{r}^{-1}(\lambda)^{2}-r e^{-\lambda r} \Delta_{r}^{-1}(\lambda) B \Delta_{r}^{-1}(\lambda), \quad \lambda \in D_{r} .
$$

Proof. For $\mu, \lambda \in D_{r}$, we have

$$
\begin{aligned}
\Delta_{r}^{-1}(\mu) & =\Delta_{r}^{-1}(\mu)\left(\lambda-A-B e^{-\lambda r}\right) \Delta_{r}^{-1}(\lambda) \\
& =\Delta_{r}^{-1}(\mu)\left(\mu-A-B e^{-\mu r}+\lambda-\mu+B e^{-\mu r}-B e^{-\lambda r}\right) \Delta_{r}^{-1}(\lambda) \\
& =\Delta_{r}^{-1}(\lambda)+\Delta_{r}^{-1}(\mu)\left(\lambda-\mu+B e^{-\mu r}-B e^{-\lambda r}\right) \Delta_{r}^{-1}(\lambda),
\end{aligned}
$$

or equivalently,

$$
\Delta_{r}^{-1}(\mu)-\Delta_{r}^{-1}(\lambda)=\Delta_{r}^{-1}(\mu)\left(\lambda-\mu+B e^{-\mu r}-B e^{-\lambda r}\right) \Delta_{r}^{-1}(\lambda) .
$$

Next, for $\lambda_{0} \in D_{r}$, we get

$$
\begin{aligned}
\Delta_{r}(\lambda) & =\lambda_{0}-A-e^{-\lambda_{0 r}} B+\left(\lambda-\lambda_{0}\right)-B e^{-\lambda r}+B e^{-\lambda_{0} r} \\
& =\Delta_{r}\left(\lambda_{0}\right)\left(I+\Delta_{r}^{-1}\left(\lambda_{0}\right)\left(\lambda-\lambda_{0}-B e^{-\lambda r}+B e^{-\lambda_{0} r}\right)\right) .
\end{aligned}
$$

Let $\lambda-\lambda_{0}$ be small enough such that

$$
\left\|\lambda-\lambda_{0}+e^{-\lambda_{0} r} B-e^{-\lambda r} B\right\| \leq \frac{1}{2\left\|\Delta_{r}^{-1}\left(\lambda_{0}\right)\right\|},
$$

which yields that $\left(I+\Delta_{r}^{-1}\left(\lambda_{0}\right)\left(\lambda-\lambda_{0}+e^{-\lambda_{0} r} B-e^{-\lambda r} B\right)\right)^{-1}$ exists and belongs to $\mathcal{L}(X)$, and $\left\|\left(I+\Delta_{r}^{-1}\left(\lambda_{0}\right)\left(\lambda-\lambda_{0}+e^{-\lambda r} B-e^{-\lambda r} B\right)\right)^{-1}\right\| \leq 2$. Therefore, we obtain

$$
\Delta_{r}^{-1}(\lambda)=\left(I+\Delta_{r}^{-1}\left(\lambda_{0}\right)\left(\lambda-\lambda_{0}+e^{-\lambda_{0} r} B-e^{-\lambda r} B\right)\right)^{-1} \Delta_{r}^{-1}\left(\lambda_{0}\right)
$$


and $\Delta_{r}^{-1}(\lambda)\|\leq 2\| \Delta_{r}^{-1}\left(\lambda_{0}\right) \|$.

On the other hand, for $\lambda$ satisfying (3.4), using the resolvent identity (3.3), we deduce that

$$
\Delta_{r}^{-1}(\lambda)-\Delta_{r}^{-1}\left(\lambda_{0}\right)=\Delta_{r}^{-1}(\lambda)\left(\lambda_{0}-\lambda+B e^{-\lambda r}-B e^{-\lambda_{0} r}\right) \Delta_{r}^{-1}\left(\lambda_{0}\right),
$$

and consequently,

$$
\begin{aligned}
\left\|\Delta_{r}^{-1}(\lambda)-\Delta_{r}^{-1}\left(\lambda_{0}\right)\right\| & \leq\left\|\Delta_{r}^{-1}(\lambda)\right\|\left\|\lambda_{0}-\lambda+B e^{-\lambda r}-B e^{-\lambda_{0} r}\right\|\left\|\Delta_{r}^{-1}\left(\lambda_{0}\right)\right\| \\
& \leq 2\left\|\Delta_{r}^{-1}\left(\lambda_{0}\right)\right\|\left\|\lambda_{0}-\lambda+B e^{-\lambda r}-B e^{-\lambda_{0} r}\right\| \stackrel{\lambda \rightarrow \lambda_{0}}{\longrightarrow} 0,
\end{aligned}
$$

which shows the continuity of $\Delta_{r}^{-1}(\cdot)$. Finally, using continuity and the resolvent identity (3.3) of $\Delta_{r}^{-1}(\cdot)$, it is easy to see the analyticity. Indeed, for $\lambda, \mu \in D_{r}$, from (3.3) it follows that

$$
\frac{\Delta_{r}^{-1}(\mu)-\Delta_{r}^{-1}(\lambda)}{\mu-\lambda}=-\Delta_{r}^{-1}(\mu)\left(I-B \frac{e^{-\mu r}-e^{-\lambda r}}{\mu-\lambda}\right) \Delta_{r}^{-1}(\lambda) .
$$

Let $\mu \rightarrow \lambda$, then using the continuity of $\Delta_{r}^{-1}(\cdot)$, we obtain

$$
\frac{d}{d \lambda} \Delta_{r}^{-1}(\lambda)=-\Delta_{r}^{-1}(\lambda)^{2}-r e^{-\lambda r} \Delta_{r}^{-1}(\lambda) B \Delta_{r}^{-1}(\lambda) .
$$

THEOREM 3.4. Let $\left\|X_{r}(t)\right\| \leq M e^{w_{1} t}, s_{0}^{r}:=\inf \left\{\operatorname{Re} \lambda \mid \lambda \in D_{r}\right.$ and $\left\|\Delta_{r}^{-1}(\lambda)\right\|<$ $+\infty\}, w_{0}^{r}:=\inf \left\{w \in R \mid\right.$ there exists $M \geq 1$ such that $\left.\left\|X_{r}(t)\right\| \leq M e^{w t}, t \geq 0\right\}$, and let $\tau_{1}>\tau_{2}>s_{0}^{r}$. Then for all $x \in X$ we have $\lim _{|s| \rightarrow \infty}\left\|\Delta_{r}^{-1}(\tau+i s) x\right\|=0$, uniformly for $\tau \in\left[\tau_{1}, \tau_{2}\right]$.

PROOF. Upon replacing $\tau_{2}$ by some large number, we may assume that $\tau_{2}>w_{0}^{r}$. By the Riemann-Lebesgue lemma, for $x \in X$ we have $\lim _{|s| \rightarrow \infty}\left\|\Delta_{r}^{-1}\left(\tau_{2}+i s\right) x\right\|=0$. Using the resolvent identity (3.3), for all $\tau \in\left[\tau_{1}, \tau_{2}\right]$, we deduce

$$
\begin{aligned}
\Delta_{r}^{-1}(\tau+i s) x= & \left\{I+\Delta_{r}^{-1}(\tau+i s)\left(\tau_{2}-\tau-B e^{-\left(\tau_{2}+i s\right) r}+B e^{-(\tau+i s) r}\right)\right\} \\
& \times \Delta_{r}^{-1}\left(\tau_{2}+i s\right) x,
\end{aligned}
$$

and hence

$$
\begin{aligned}
\left\|\Delta_{r}^{-1}(\tau+i s) x\right\| \leq & \left\{1+\left\|\Delta_{r}^{-1}(\tau+i s)\right\|\left(\left|\tau_{2}-\tau\right|+\|B\|\left|e^{-\left(\tau_{2}+i s\right) r}-e^{-(\tau+i s) r}\right|\right)\right\} \\
& \times\left\|\Delta_{r}^{-1}\left(\tau_{2}+i s\right) x\right\| \\
\leq & \left\{1+\sup _{\tau \in\left[\tau_{1}, \tau_{2}\right]}\left\|\Delta_{r}^{-1}(\tau+i s)\right\|\left(\left|\tau_{2}-\tau\right|+\|B\|\left(e^{-\tau_{2} r}+e^{-\tau r}\right)\right)\right\} \\
& \times\left\|\Delta_{r}^{-1}\left(\tau_{2}+i s\right) x\right\| \\
\rightarrow & 0 \quad(|s| \rightarrow \infty),
\end{aligned}
$$

uniformly for $\tau \in\left[\tau_{1}, \tau_{2}\right]$. 
THEOREM 3.5. Let $X_{r}(t)$ be the fundamental operator family of system (1.1). Then for all $x \in X$ and $\omega>s_{0}^{r}$ we have

$$
X_{r}(t) x=\frac{1}{2 \pi i}(C, 1) \int_{\operatorname{Re} \lambda=w} e^{\lambda t} \Delta_{r}^{-1}(\lambda) x d \lambda,
$$

where $(C, 1)$ denotes convergence of the integral in the Césaro mean, that is,

$$
(C, 1) \int_{-\infty}^{+\infty} f(s) d s:=\lim _{t \rightarrow+\infty} \frac{1}{t} \int_{0}^{t} \int_{-\tau}^{+\tau} f(s) d s d \tau .
$$

PROOF. For $w>w_{0}^{r}$, see the definition in Theorem 3.4. From Fejer's theorem [17], it follows that

$$
e^{-w t} X_{r}(t) x=\frac{1}{2 \pi}(C, 1) \int_{R} e^{i s t} e^{-\widehat{w \cdot} X_{r}(\cdot)} x(s) d s,
$$

where $\wedge$ denotes the Fourier transform. On the other hand, by Theorem 2.2, we have

$$
\Delta_{r}^{-1}(w+i s) x=\int_{0}^{\infty} e^{-(w+i s) t} X_{r}(t) x d t=e^{-\widehat{w \cdot} X_{r}(\cdot)} x(s) .
$$

Therefore, we have

$$
\begin{aligned}
X_{r}(t) x & =\frac{e^{w t}}{2 \pi}(C, 1) \int_{R} e^{i s t} \Delta_{r}^{-1}(w+i s) x d s \\
& =\frac{1}{2 \pi i}(C, 1) \int_{R e \lambda=w} e^{\lambda t} \Delta_{r}^{-1}(\lambda) x d \lambda .
\end{aligned}
$$

Finally, for general $w>s_{0}^{r}$, using Theorem 3.4 and Cauchy's theorem to shift the path of the integral to a vertical line to the right of $\omega_{0}^{r}$, we obtain

$$
(C, 1) \int_{\operatorname{Re} \lambda=w} e^{\lambda t} \Delta_{r}^{-1}(\lambda) x d \lambda=(C, 1) \int_{\operatorname{Re} \lambda=w_{0}^{\prime}} e^{\lambda t} \Delta_{r}^{-1}(\lambda) x d \lambda .
$$

THEOREM 3.6. If there exists an $w^{\prime}>w_{0}^{r}$ and $M_{r}>0$ such that

$$
\int_{R}\left\|\Delta_{r}^{-1}\left(w^{\prime}+i s\right) x\right\|^{2} d s<M_{r}\|x\|^{2}, \quad x \in X,
$$

and

$$
\int_{R}\left\|\Delta_{r}^{-1}\left(w^{\prime}+i s\right)^{*} x^{*}\right\|^{2} d s<M_{r}\|x\|^{2}, \quad x^{*} \in X^{*},
$$

then $w_{0}^{r}=s_{0}^{r}$.

Suppose that (3.5)-(3.6) are satisfied uniformly for $r \in\left[0, r_{0}\right]$ and $\sup \left\{\left\|\Delta_{r}^{-1}(\lambda)\right\|:\right.$ $\operatorname{Re} \lambda>0\} \leq M$, uniformly for $r \in\left[0, r_{0}\right]$. Then $X_{r}(t)$ is uniformly exponentially stable for $r \in\left[0, r_{0}\right]$. 
PROOF. It suffices to prove $s_{0}^{r} \geq w_{0}^{r}$. Indeed, for any $\varepsilon>0$, from (3.3), we deduce

$$
\begin{aligned}
\Delta_{r}^{-1}\left(s_{0}^{r}+\varepsilon+i s\right)= & \Delta_{r}^{-1}\left(w^{\prime}+i s\right)+\Delta_{r}^{-1}\left(s_{0}^{r}+\varepsilon+i s\right) \\
& \times\left(w^{\prime}-s_{0}^{r}-\varepsilon+B e^{-\left(s_{0}^{\prime}+\varepsilon+i s\right) r}-B e^{-\left(w^{\prime}+i s\right) r}\right) \Delta_{r}^{-1}\left(w^{\prime}+i s\right),
\end{aligned}
$$

and hence

$$
\begin{aligned}
(C, 1) & \int_{R} e^{i s t} \Delta_{r}^{-1}\left(s_{0}^{r}+\varepsilon+i s\right) x d s \\
= & (C, 1) \int_{R} e^{i s t} \Delta_{r}^{-1}\left(w^{\prime}+i s\right) x d s \\
& +(C, 1) \int_{R} e^{i s t} \Delta_{r}^{-1}\left(s_{0}^{r}+\varepsilon+i s\right)\left(w^{\prime}-s_{0}^{r}-\varepsilon\right. \\
& \left.+B e^{-\left(s_{0}^{\prime}+\varepsilon+i s\right) r}-B e^{-\left(w^{\prime}+i s\right) r}\right)^{-1} \Delta_{r}^{-1}\left(w^{\prime}+i s\right) x d s .
\end{aligned}
$$

On the other hand, we estimate

$$
\left\|\frac{1}{2 \pi}(C, 1) \int_{R} e^{i s t} \Delta_{r}^{-1}\left(w^{\prime}+i s\right) x d s\right\| \leq\left\|e^{-w^{\prime} t} X_{r}(t) x\right\| \leq M\|x\|,
$$

and from (3.5) and (3.6) we estimate

$$
\begin{aligned}
\|(C, 1) & \int_{R} e^{i s t} \Delta_{r}^{-1}\left(s_{0}^{r}+\varepsilon+i s\right)\left(w^{\prime}-s_{0}^{r}-\varepsilon+B e^{-\left(s_{0}^{\prime}+\varepsilon+i s\right) r}-B e^{-\left(w^{\prime}+i s\right) r}\right) \\
& \times \Delta_{r}^{-1}\left(w^{\prime}+i s\right) x d s \| \\
= & \sup _{\left\|x^{*}\right\| \leq 1} \mid(C, 1) \int_{R} e^{i s t} x^{*}\left\{\Delta_{r}^{-1}\left(s_{0}^{r}+\varepsilon+i s\right)\right. \\
& \left.\times\left(w^{\prime}-s_{0}^{r}-\varepsilon+B e^{-\left(s_{0}^{\prime}+\varepsilon+i s\right) r}-B e^{-\left(w^{\prime}+i s\right) r}\right) \Delta_{r}^{-1}\left(w^{\prime}+i s\right) x\right\} d s \mid \\
\leq & \sup _{\left\|x^{*}\right\| \leq 1} \int_{R}\left\|\Delta_{r}^{-1}\left(s_{0}^{r}+\varepsilon+i s\right)^{*} x^{*}\right\| \\
& \times\left\|\left(w^{\prime}-s_{0}^{r}-\varepsilon+B e^{-\left(s_{0}^{\prime}+\varepsilon+i s\right) r}-B e^{-\left(w^{\prime}+i s\right) r}\right) \Delta_{r}^{-1}\left(w^{\prime}+i s\right) x\right\| d s \\
\leq & \sup _{\left\|x^{*}\right\| \leq 1}\left\{\int_{R}\left\|\Delta_{r}^{-1}\left(s_{0}^{r}+\varepsilon+i s\right)^{*} x^{*}\right\|^{2} d s\right\}^{1 / 2} \\
& \times\left(\int_{R} \|\left(w^{\prime}-s_{0}^{r}-\varepsilon+B e^{-\left(s_{0}^{\prime}+\varepsilon+i s\right) r}-B e^{-\left(w^{\prime}+i s\right) r}\left\|^{2}\right\| \Delta_{r}^{-1}\left(w^{\prime}+i s\right) x \|^{2} d s\right)^{1 / 2}\right. \\
\leq & M\left(\left|w^{\prime}-s_{0}^{r}-\varepsilon\right|+\|B\|\left(e^{\left|s_{0}^{\prime}+\varepsilon\right| r_{0}}+e^{\left.\left|w^{\prime}\right| r_{0}\right)}\right)\left(\int_{R}\left\|\Delta_{r}^{-1}\left(w^{\prime}+i s\right) x\right\|^{2} d s\right)^{1 / 2}\right. \\
\leq & M^{\prime}\|x\| .
\end{aligned}
$$


Therefore, using Theorem 3.5 and Equations (3.7)-(3.9), we obtain

$$
\begin{aligned}
\left\|X_{r}(t) x\right\| & =\left\|\frac{1}{2 \pi}(C, 1) \int_{R} e^{\left(s_{0}^{r}+\varepsilon+i s\right) t} \Delta_{r}^{-1}\left(s_{0}^{r}+\varepsilon+i s\right) x d s\right\| \\
& =\left\|\frac{e^{\left(s_{0}^{\prime}+\varepsilon\right) t}}{2 \pi}(C, 1) \int_{R} e^{i s t} \Delta_{r}^{-1}\left(s_{0}^{r}+\varepsilon+i s\right) x d s\right\| \\
& \leq M_{\varepsilon} e^{\left(s_{0}^{r}+\varepsilon\right) t}\|x\|,
\end{aligned}
$$

which, observing the arbitrariness of $\varepsilon>0$, completes the proof.

\section{Acknowledgements}

This research was partially supported by the National Natural Science Foundation of China and the Postdoctoral Science Foundation of China.

\section{References}

[1] G. Avalos, I. Lasiecka and R. Rebarber, "Lack of time-delay robustness for stabilization of a structural acoustics model", SIAM J. Control Optim. 37 (1999) 1394-1428.

[2] A. Bátkai and S. Piazzera, "Partial differential equations with unbounded operators in the delay term", Tübinger Berchte Funktionalanalysis 9 (2000) 69-83.

[3] C. Corduneanu, "Some differential equations with delay", in Proceedings, Equadiff. 3, (Czechoslovak Conference on Differential Equations and Applications), (Springer, London, 1972), 105-114.

[4] R. Datko, "Not all feedback stabilized systems are robust with respect to small delays in their feedback", SIAM J. Control Optim. 26 (1988) 697-7113.

[5] R. Datko, J. Lagnese and M. P. Polis, "An example on the effect of time delays in boundary feedback of wave equations", SIAM J. Control Optim. 24 (1986) 69-83.

[6] K. J. Engel and R. Nagel, One-parameter semigroups for linear evolution equations, Graduate Texts in Math. 194 (Springer, New York, 1999).

[7] 3. K. Hale and S. M. Verduyn Lunel, "Effects of small delays on stability and control", Oper. Theory Adv. Appl. 122 (2001) 275-301.

[8] F. L. Huang, "Characteristic conditions for exponential stability of linear dynamical systems in Hilbert spaces", Ann. Differential Equations 1 (1985) 45-53.

[9] F. L. Huang, "On the stability with respect to small delays for linear differential equations on Banach spaces", Chinese J. Math. 6 (1986) 183-191, (in Chinese).

[10] X. J. Li and K. S. Liu, "The effect of small delays in the feedbacks on boundary stabilization", Sci. China Ser. A 36 (1993) 1453-1443.

[11] J. Liang and T. J. Xiao, "Functional differential equations with infinite delay in Banach spaces", Internat. J. Math. Math. Sci. 14 (1991) 1331-1341.

[12] J. Liang and T. J. Xiao, "Exponential stability for abstract autonomous functional differential equations with infinite delay", Internat. J. Math. Math. Sci. 21 (1998) 255-260.

[13] H. Logemann, "Destabilizing effect of small delays on feedback-controlled descriptor systems", Linear Algebra Appl. 272 (1998) 131-153. 
[14] H. Logemann and R. Rebarber, "The effect of small delays on the closed-loop stability of boundary control systems", Math. Control Signals Systems 9 (1996) 123-151.

[15] H. Logemann, R. Rebarber and G. Weiss, "Conditions for robustness and nonrobustness of the stability of feedback systems with respect to small delays in the feedback loop", SIAM J. Control Optim. 37 (1996) 572-600.

[16] R. Rebarber and S. Townley, "Robustness with respect to delays for exponential stability of distributed parameter systems", SIAM J. Control Optim. 37 (1998) 230-214.

[17] J. M. A. M. van Neerven, The asymptotic behaviour of semigroups of linear operators, Operator Theory: Advances and Applications 88 (Birkhäuser Verlag, Basel, 1996).

[18] Q. Zhang, Strongly continuous semigroups of linear operator, (in Chinese) (Huangzhong University of Science and Technology Press, Wuhan, 1994). 